\title{
Immune Response of BALB/c Mice toward Putative Calcium Transporter Recombinant Protein of Trichomonas vaginalis
}

\author{
Tahali Mendoza-Oliveros¹, Victor Arana-Argáez², Leidi C. Alvaréz-Sánchez³ , Julio Lara-Riegos', \\ María Elizbeth Alvaréz-Sánchez ${ }^{4}$, Julio C. Torres-Romero"* \\ 'Laboratorio de Bioquímica y Genética Molecular, Facultad de Química de la Universidad Autónoma de Yucatán, Mérida 97069, México; \\ ²Laboratorio de Farmacología, Facultad de Química de la Universidad Autónoma de Yucatán, Mérida 97069, México; ${ }^{3}$ Laboratorio de Virología, \\ Centro de Investigaciones Regionales "Dr. Hideyo Noguchi" de la Universidad Autónoma de Yucatán, Mérida 97069, México; "Posgrado en \\ Ciencias Genómicas, Universidad Autónoma de la Ciudad de México (UACM), Ciudad de México 03100, México
}

\begin{abstract}
Trichomoniasis is a common sexually transmitted infection caused by Trichomonas vaginalis, which actually does not exist a vaccine for control or prevention. Thus, the identification of new and potent immunogens in $T$. vaginalis, which can contribute to the development of a vaccine against this parasite, is necessary. Therefore, the aim of this work was to evaluate the potential of a recombinant Transient Receptor Potential-like channel of T. vaginalis (TvTRPV), as a promising immunogen in BALB/c mice. First, TVTRPV was cloned and expressed as a recombinant protein in Escherichia coli BL21 cells and purified by nickel affinity. Next, BALB/c mice were immunized and the antibody levels in mice serum and cytokines from the supernatant of macrophages and from co-culture systems were evaluated. Recombinant TvTRPV triggered high levels of specific total lgG in sera from the immunized mice. Also, a statistically significant increase of cytokines: IL-1 $\beta$, IL-6, and TNF- $\alpha$ after stimulation with the corresponding antigens in vitro, was identified. Moreover, co-cultures using $\mathrm{CD}^{+}{ }^{+} \mathrm{T}$ cells from immunized mice were able to identify higher levels of IL-10 and IFN- $\gamma$. These results were useful to validate the immunogenicity of TVTRPV in BALB/c mice, where IL-10-IFN- $\gamma$-secreting cells could play a role in infection control, supporting the potential of TVTRPV as a promising target for vaccine against T. vaginalis.
\end{abstract}

Key words: Trichomonas vaginalis, TRPV channel, recombinant protein, immune response, BALB/c mouse

Trichomoniasis is the most common non-viral sexually transmitted infection in the world, caused by the protozoan parasite Trichomonas vaginalis with an incidence of 276 million new cases each year [1]. Trichomoniasis can increase the risk of getting or spreading other sexually transmitted infections, such as human immunodeficiency virus (HIV) or human papilloma virus (HPV) $[2,3]$. With an increasing incidence of infection and a possible non-effective treatment, the prevention of this disease with a vaccine would clearly be desirable [4]. Until now, work on the development of $T$. vaginalis vaccines is still mainly focused on the screening of parasite-derived immunogens capable of inducing both humoral and cellular immune responses $[5,6]$.

\footnotetext{
- Received 11 August 2018, revised 4 December 2018, accepted 23 December 2018. *Corresponding author (jullio.torres@correo.uady.mx) (c) 2019, Korean Society for Parasitology and Tropical Medicine This is an Open Access article distributed under the terms of the Creative Commons Attribution Non-Commercial License (http://creativecommons.org/licenses/by-nc/4.0) which permits unrestricted non-commercial use, distribution, and reproduction in any medium, provided the original work is properly cited.
}

Some microbial surface proteins are considered as potential immunogens. Specifically, ion transporters from diverse microorganisms have been reported that they may be suitable targets for development of live attenuated vaccines, exhibiting superior protective immunity compared with commercial vaccines $[7,8]$. In addition, it has been described that components of ionic transporters can induce antibodies that promote specific immune responses rather than inhibiting ionic transport $[9,10]$.

Transient Receptor Potential (TRP) family of ion channels serve as cellular sensors for a wide spectrum of physical and chemical stimuli [11]. Prole and Taylor examined several protozoan parasite genomes and found that only T. vaginalis had a unique homologue of TRPV channels (TVTRPV), which was predicted to exist on membrane surface of this parasite [12]. Some members of the TRP family have been shown to be associated with inflammatory processes and immune responses [13]. Recently, it was identified that immunization of mice with a variety of antigens expressing TRPA1 channels resulted 
in the generation of monoclonal antibodies that could act as selective antagonists [14].

Taking into account the immunogenic potential described in some ionic transporters, the association of some members of the TRP family with immune responses, the possible location of TvTRPV in T. vaginalis, as well as its important role in different cellular processes, TvTRPV shows potential to be an immunogenic protein and the present study reports the humoral and cellular immune response of $\mathrm{BALB} / \mathrm{c}$ mice immunized with the recombinant TVTRPV protein from $T$. vaginalis.

For this work, total RNA isolation from T. vaginalis trophozoites and cDNA synthesis were carried out as reported previously [15]. TvTRPV gene (Genbank Accession: XP_001296819) was amplified from cDNA by PCR using specific primers and cloned into the pCold-II (Takara, Otsu, Shiga, Japan) prokaryotic expression vector, by insertion at BamHI and HindIII restriction sites. Transformed E. coli BL21 (DE3) (Promega, Madison, Wisconsin, USA) cells were grown in $\mathrm{LB}$ at $37^{\circ} \mathrm{C}$ and protein expression was induced at OD600 of 0.4 with $1 \mathrm{mM}$ isopropyl- $\beta$-D-1-thiogalactoside (IPTG) (Promega, Madison, Wisconsin, USA) for $24 \mathrm{hr}$. Culture conditions were optimized to achieve a recombinant protein concentration equal to approximately $1 \mathrm{mg} / \mathrm{ml}$ culture medium. After induction, the protein was purified from the soluble fraction using His-spin protein miniprep kit (Zymo Research, Irvine, California, USA) following the manufacturer's instructions, except that all steps were performed at $4^{\circ} \mathrm{C}$ and increased to 5 washes prior the elution step.

Once obtained the recombinant protein, 30 male BALB/c mice were randomly divided in groups of 5 and were immunized subcutaneously: 3 experimental groups were immunized with recombinant TvTRPV at doses of 50, 100, and 200 $\mu \mathrm{g} / \mathrm{kg}$ (TvTRPV50, TvTRPV100, and TvTRPV200, respectively), and 2 control groups, 1 immunized with Incomplete Freund's Adjuvant (IFA) (Santa Cruz Biotechnology, Dallas, Texas, USA) and a second unimmunized control (C-). Two booster injections were given in 2-week interval.

Blood was collected from the mice by tail vein puncture before each immunization and to analyze the humoral response to anti-recombinant TvTRPV we measured antigen-specific IgG antibody levels in sera by enzyme-linked immunosorbent assay (ELISA). Microtiter plates were coated with the recombinant protein $(1 \mu \mathrm{g} / \mathrm{ml}, 100 \mu \mathrm{l} /$ well $)$ overnight at $4^{\circ} \mathrm{C}$ in carbonate buffer (0.05 M carbonate-bicarbonate, $\mathrm{pH}$ 9.6). The non-specific sites were blocked with blocking buffer (Peprot- ech, London, UK) for $2 \mathrm{hr}$ at room temperature. Serum samples from mice were diluted (1:25) in diluent buffer $1 \mathrm{X}$ (Peprotech), were loaded to each well and then incubated for $2 \mathrm{hr}$ at room temperature. Anti-mouse IgG conjugated to HRP (Santa Cruz Biotechnology) were diluted (1:1,500), added to each well and incubated for $2 \mathrm{hr}$ at room temperature. Later, ABTS was added and incubated for $30 \mathrm{~min}$ in the dark at room temperature. Immediately, the absorbance value of wells was read using an ELISA reader (Bio-Rad iMark, California, USA) at a wavelength of $450 \mathrm{~nm}$. Triplicate biological replicates of samples from each experimental group were evaluated.

We also examined whether recombinant TvTRPV induces macrophages to secrete proinflammatory cytokines by antigen stimulation peritoneal macrophages (MØs), which were isolated from mice as described previously [16]. MØs were seeded at $1 \times 10^{5} \mathrm{cells} / \mathrm{ml} /$ well in 24-well plate and incubated with recombinant TvTRPV protein at 1, 10, and $100 \mu \mathrm{g} / \mathrm{ml}$ in complete medium (DMEM supplemented with 10\% FBS and 1\% penicillin-streptomycin) for $48 \mathrm{hr}\left(37^{\circ} \mathrm{C}, 5 \% \mathrm{CO}_{2}\right)$. Macrophages without treatment were used as negative control (C-) and activated macrophages with LPS $(1 \mu \mathrm{g} / \mathrm{ml})$ were employed as a positive control. The maturation of macrophages was confirmed by microscopic monitoring the change in shape by the increment of the cytoplasmic volume and granularity.

We also evaluated the antigenic specific cellular immune response using $\mathrm{CD}^{+}{ }^{+} \mathrm{T}$ cells which were co-incubated with mice macrophages treated with recombinant TvTRPV. For this coculture system, mice were euthanized 4 weeks after the last boost and $\mathrm{CD}^{+}{ }^{+} \mathrm{T}$-cells were isolated from mouse spleens as described previously [17]. Cell suspension was prepared in 90 $\mu$ of isolation buffer per $1 \times 10^{7}$ total cells. Resuspended CD4 ${ }^{+}$ T-cells were purified by negative selection by using $\mathrm{CD} 4^{+} \mathrm{T}$ cells isolation kit (Miltenyi Biotec, Bergisch Gladbach, Germany) according to the manufacturer's instructions. $\mathrm{CD} 4^{+} \mathrm{T}$ cells were incubated with macrophages previously obtained, in the following proportions: $1 \mathrm{CD} 4^{+} \mathrm{T}$ cell for each 2 macrophages (1:2). Cells were grown in this co-culture system for $72 \mathrm{hr}$ $\left(37^{\circ} \mathrm{C}, 5 \% \mathrm{CO}_{2}\right)$ in a 24 -well plate for IFN- $\gamma$ production.

Cell culture medium of treated and activated macrophages (for IL-1 $\beta$, IL- 6 , and TNF- $\alpha$ detection) and co-culture systems of macrophages and $\mathrm{CD} 44^{+} \mathrm{T}$ cells (for IFN- $\gamma$, and $1 \mathrm{~L}-10$ evaluation) were analyzed through commercial ELISA kits according to the manufacturer's instructions (Peprotech). Microtiter plates were coated with capture antibody $(1 \mu \mathrm{g} / \mathrm{ml}, 100 \mu \mathrm{l} /$ well $)$ overnight at $4^{\circ} \mathrm{C}$ for IL-1 $\beta$, IL-6, TNF- $\alpha$, IFN- $\gamma$, and $1 \mathrm{~L}-10$ evaluation. 
The non-specific sites were blocked with blocking buffer (1\% BSA in PBS) for $1 \mathrm{hr}$ at room temperature. Serial dilutions of recombinant cytokines were used as standard curve in each case, as recommended by the manufacturer. The cytokines present in the supernatants were detected by adding biotinylated avidin-HRP conjugated to detection antibody (Peprotech) and incubated overnight. The absorbance values at $490 \mathrm{~nm}$ were determined using an ELISA plate reader (Bio-Rad iMark) and the cytokines concentration was determined by comparison of the absorbance values in the corresponding standard curve.

The present study was approved by the ethics committee of the Campus of Agricultural and Biological Sciences (CB-CCBAM-2016-005) at Autonomous University of Yucatan, Mexico, and conducted according to the guidelines outlined in the declaration of Helsinki.

A $711 \mathrm{bp}$ segment corresponding to the TvTRPV gene was amplified from cDNA, cloned into pCold-II expression vector and expressed as a polyhistidine fusion protein, observed as a band about $27.4 \mathrm{kDa}$ in an SDS-PAGE only from soluble fraction after $24 \mathrm{hr}$ of induction. The amount of purified recombinant TvTRPV was estimated as $0.7 \mathrm{mg} / \mathrm{ml}$ (Fig. 1).

The humoral response to anti-recombinant TvTRPV show that antigen-specific IgG antibody response was significantly higher in the mice immunized with recombinant TvTRPV (TvTRPV50, TvTRPV100, and TvTRPV200 groups) at 2 weeks after the first immunization, compared to the control groups (IFA and C-) (Table 1).

We detected high levels of IgG in mice immunized with recombinant TvTRPV (TvTRPV50, TvTRPV100, and TvTRV200 groups), which showed a concentration-dependent increase following booster immunizations (week 4 and 6, respectively). Although TvTRPV50, TvTRPV100, and TvTRPV200 groups showed a gradual increase in the production of IgG antibodies at the different times evaluated, we did not observe statistical differences between the 3 groups, except for TvTRPV200 group that showed a significant increase $(P<0.05)$ after the first and second booster immunizations with respect to the IgG levels detected in IFA group (Table 1). These results suggest that in $\mathrm{BALB} / \mathrm{c}$ mice, recombinant TvTRPV stimulates a high IgG response. The data also suggest that the booster immunization of the highest dose enhances the immune response induced by this recombinant protein.

We also examined whether recombinant TvTRPV induces macrophages to secrete proinflammatory cytokines. For this purpose, macrophages were stimulated in vitro with 1,10 , and $100 \mu \mathrm{g} / \mathrm{ml}$ of recombinant protein. LPS-stimulated macrophages were used as a positive control, and unstimulated macrophages as negative control. The obtained results showed that macrophages incubated with different doses of recombinant TvTRPV stimulated the production of IL-1 $\beta$, IL- 6 , and TNF- $\alpha$, showing a concentration-dependent increase of the recombinant TvTRPV in the secretion of these proinflammatory cytokines. The levels of IL-1 $\beta$ and IL- 6 detected in recombinant TvTRPV-stimulated macrophages displayed a significant increase with respect to the negative control and the levels of TNF- $\alpha$ detected in macrophages stimulated with the highest dose of the recombinant protein $(100 \mu \mathrm{g} / \mathrm{ml})$ presented statically significant difference with respect to the unimmunized

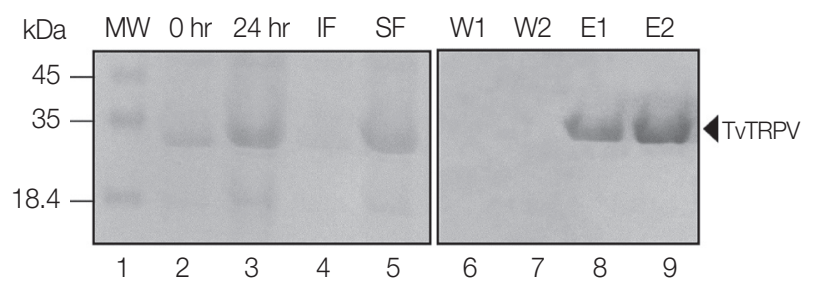

Fig. 1. Prokaryotic expression and purification of recombinant TVTRPV. 0 hr, E. coli BL21 not induced; 24 hr, induction time after adding 1 mM IPTG; IF, insoluble fraction of induced cells; SF, soluble fraction of induced cells; W1 and W2, washings E1 and E2, TVTRTPV protein obtained by elution; MW, molecular weight marker.

Table 1. Levels of IgG in the sera of mice immunized with recombinant TVTRPV protein

\begin{tabular}{lcccc}
\hline \multirow{2}{*}{ Groups } & \multicolumn{3}{c}{ lgG level $(450 \mathrm{~nm})$} \\
\cline { 2 - 5 } & Week 0 & Week 2 & Week 4 & Week 6 \\
\hline C (-) & $0.22 \pm 0.02$ & $0.22 \pm 0.03$ & $0.23 \pm 0.03$ & $0.21 \pm 0.02$ \\
Ady & $0.20 \pm 0.02$ & $0.21 \pm 0.02$ & $0.31 \pm 0.05$ & $0.31 \pm 0.05$ \\
TVTRPV50 & $0.22 \pm 0.04$ & $0.57 \pm 0.11^{*}$ & $0.56 \pm 0.10^{*}$ & $0.71 \pm 0.19^{\star *}$ \\
TVTRPV100 & $0.22 \pm 0.03$ & $0.57 \pm 0.10^{*}$ & $0.56 \pm 0.11^{\star}$ & $0.68 \pm 0.15^{\star \star}$ \\
TVTRPV200 & $0.21 \pm 0.04$ & $0.64 \pm 0.10^{\star \star}$ & $0.66 \pm 0.17^{\star \star}$ & $0.77 \pm 0.15^{\star \star}$ \\
\hline
\end{tabular}

${ }^{\star} P<0.05$; ${ }^{\star \star} P<0.01$; compared with the controls (week 0 ). 

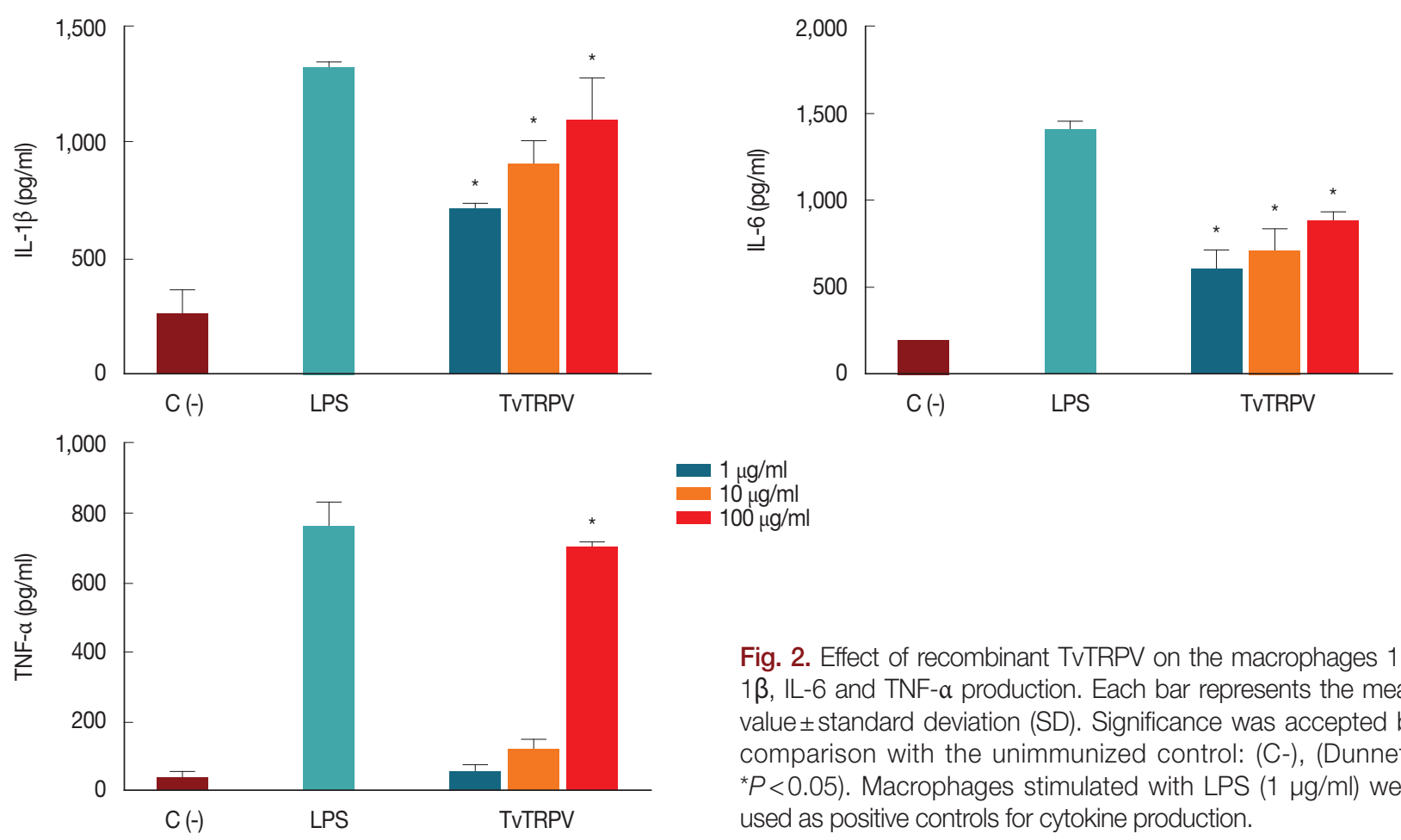

Fig. 2. Effect of recombinant TVTRPV on the macrophages $1 \mathrm{~L}-$ $1 \beta, I L-6$ and TNF- $\alpha$ production. Each bar represents the mean value \pm standard deviation (SD). Significance was accepted by comparison with the unimmunized control: (C-), (Dunnett, $\left.{ }^{\star} P<0.05\right)$. Macrophages stimulated with LPS $(1 \mu \mathrm{g} / \mathrm{ml})$ were used as positive controls for cytokine production.
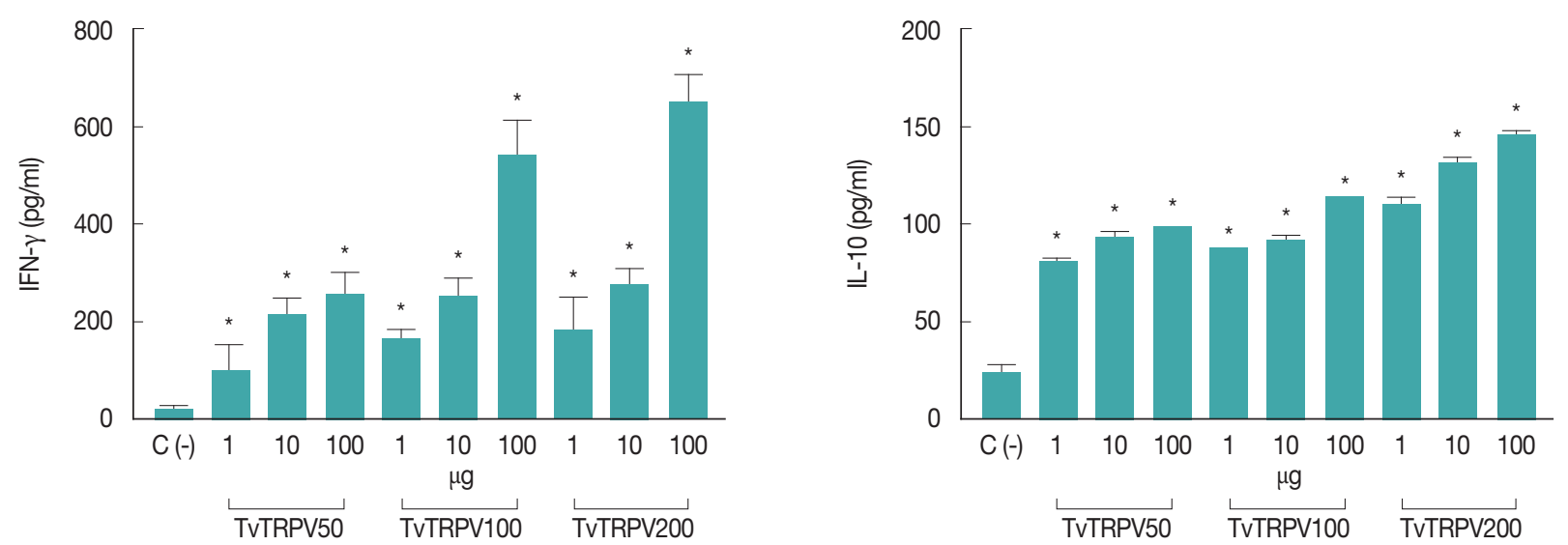

Fig. 3. IFN- $\gamma$ and IL-10 levels detected in co-culture system. Purified CD4+ T cells from immunized mice with different doses of recombinant TVTRPV (TVTRPV50, TVTRPV100, and TVTRPV200) stimulated with macrophages treated with different doses of recombinant TVTRPV $(1,10$, and $100 \mu \mathrm{g})$. Co-culture systems were set up with 1:2 CD4+ T-cell:MØ. Each bar represents the mean value \pm standard deviation (SD). Significance was accepted by comparison with $\mathrm{CD}^{+} \mathrm{T}$ cells stimulated with macrophages without treatment: $\mathrm{C}(-)$, (Dunnett, $\left.{ }^{\star} P<0.05\right)$.

control (C-) (Fig. 2). These results indicate that the recombinant TvTRPV is recognized by antigen-presenting cells, such as macrophages, with the subsequent stimulation in the secretion of proinflammatory cytokines.

On the other hand, when the obtained $\mathrm{CD} 4{ }^{+} \mathrm{T}$ cells were co-incubated with mice macrophages treated with recombinant TvTRPV, they secreted detectable levels of IFN- $\gamma$ and IL10. In vitro treatment of $\mathrm{CD} 4^{+} \mathrm{T}$ cells from recombinant
TvTRPV-immunized mice stimulated with macrophages previously treated with different doses $(1,10$, and $100 \mu \mathrm{g} / \mathrm{ml})$ of this recombinant protein triggered significantly higher amounts of IFN- $\gamma$ when compared to the level of this cytokine produced by $\mathrm{CD} 4^{+} \mathrm{T}$ cells stimulated with macrophages without treatment (C-) (Fig. 3). Also, the levels of IL-10 detected in $\mathrm{CD} 4^{+} \mathrm{T}$ cells from recombinant TvTRPV-immunized mice with the highest doses (100 and $200 \mu \mathrm{g} / \mathrm{kg}$ ) and that were stimulated 
with macrophages treated with $100 \mu \mathrm{g} / \mathrm{ml}$ of recombinant TvTRPV were significantly higher in comparison with those produced by $\mathrm{CD} 4^{+} \mathrm{T}$ cells co-cultured with untreated macrophages (Fig. 3). These results showed that recombinant TvTRPV was able to induce a broad range of cytokines such as of IL-1 $\beta$, IL-6, TNF- $\alpha$, IL-10, and IFN- $\gamma$. These latter deserve particular mention, since were produced by $\mathrm{CD} 4^{+} \mathrm{T}$ cells, a regulatory population previously described and named IL-10IFN- $\gamma$-secreting $\mathrm{CD}^{+}{ }^{+} \mathrm{T}$ cells.

To evaluate the immunological potential of a protein, the study of the humoral immune response is one of the most important parameters. In this work, recombinant TvTRPV derived from T. vaginalis triggered the production of IgG antibodies against this protein. Our results showed significantly higher IgG levels in mice immunized with the highest dose of recombinant TvTRPV protein $(200 \mu \mathrm{g} / \mathrm{kg})$ in all the times evaluated. In addition, the low and intermediate doses (50 and $100 \mu \mathrm{g} /$ $\mathrm{kg}$ ) were capable to stimulate the production of IgG antibodies. These results are consistent with previous reports, in which T. vaginalis isolates were used to produce whole-cell vaccines and higher levels of IgG were detected in serum of mice after vaccination compared with controls $[18,19]$.

Although the protective role of antibodies during T. vaginalis infection is unclear and there is little evidence that antibodies are effective in the elimination of parasites in vivo [1], a previous report in which mice were immunized with low and high doses of recombinant $\alpha$-actinin subunits, showed IgG antibody titers related to protective effect. These results suggest that a protective effect against $T$. vaginalis could possibly be related, at least in part, to a humoral response [5]. However, further studies are required to determine if the antibodies produced against recombinant TvTRPV provide a protective effect against the infection caused by T. vaginalis.

On the other hand, different components of the innate immune response could be participating in defense mechanisms against T. vaginalis. In trichomoniasis it has been suggested that TNF- $\alpha$, IL-1 $\beta$ (known chemotactic mediators involved in inflammation), and IL-6 produced by macrophages stimulated with T. vaginalis may play a crucial role in vivo as mediators of neutrophilic tissue recruitment at the infected sites in women [20]. In this study it was demonstrated that recombinant TvTRPV was able to stimulate effector functions of murine macrophages, finding a significant increase in the levels of IL$1 \beta$, IL- 6 and TNF- $\alpha$, compared with non-stimulated macrophages, which, interestingly, coincides with the reports men- tioned above, suggesting that TvTRPV protein from T. vaginalis could play a critical role in the initiation of the inflammatory process during the infection establishment.

These results are in agreement with previous reports in which both Trypanosoma cruzi and Leishmania major have been shown to stimulate the increased secretion of cytokines such as TNF- $\alpha$, IL-6, among others, from mice macrophages [21,22]. Besides humoral and innate response, the cellular immune response is also an important index for detection of immunogenic potential of proteins. For this purpose, we determined the level of Th1/Th2 cell related cytokines (IFN- $\gamma$ and IL-10) in the supernatant of co-culture systems using $\mathrm{CD}^{+} \mathrm{T}$ cells from spleen following immunization and re-stimulation in vitro. $\mathrm{CD}^{+}$T-helper cells may play a prominent role in parasite elimination, parasite persistence or pathological events during T. vaginalis infection $[1,23]$.

Our findings showed that recombinant TvTRPV-activated macrophages promote the secretion of significantly higher levels of IFN- $\gamma$ and IL-10 in co-cultures with CD4 ${ }^{+} \mathrm{T}$ cells from mice immunized with this recombinant protein, than those from the unimmunized control. It has been demonstrated that IFN- $\gamma$ increase macrophage-mediated cytotoxicity against T. vaginalis and also it is suggested that participate in the elimination or suppression of proliferation of T. vaginalis by stimulation of nitric oxide production as one of the effectors [24], while IL-10 is known to initiate an anti-inflammatory feedback loop by the cooperative action with other immune cells [25].

The obtained results demonstrate that recombinant TVTRPV after being recognized by macrophages and presented to the $\mathrm{T}$ cells clones producing IFN- $\gamma$ and IL-10, suggesting that cellular response against this protein could be related to a protective immunity against the protozoan parasite. The characteristics and mechanisms of antibodies and cytokines induced by this protein would provide valuable knowledge for alternative therapeutics against trichomoniasis. Therefore, further experiments are necessary in order to elucidate the molecular mechanisms by which TvTRPV exerts a cellular and humoral response in mice and if these have a protective effect on the infection caused by T. vaginalis.

\section{ACKNOWLEDGMENT}

This work was undertaken as part of a research project supported by Grant 237990 (to J.C. Torres-Romero) from Consejo Nacional de Ciencia y Tecnología (CONACYT), México. T. Ol- 
iveros-Mendoza was a scholarship recipient from CONACYT.

\section{CONFLICTS OF INTEREST}

The authors declare that they have no conflicts of interest.

\section{REFERENCES}

1. Menezes CB, Frasson AP, Tasca T. Trichomoniasis - are we giving the deserved attention to the most common non-viral sexually transmitted disease worldwide? Microb Cell 2016; 3: 404-419.

2. Kissinger P, Adamski A. Trichomoniasis and HIV interactions: a review. Sex Transm Infect 2013; 89: 426-433.

3. Lazenby GB, Taylor PT, Badman BS, McHaki E, Korte JE, Soper DE, Young Pierce J. An association between Trichomonas vaginalis and high-risk human papillomavirus in rural Tanzanian women undergoing cervical cancer screening. Clin Ther 2014; 36: 38-45.

4. Cudmore SL, Delgaty KL, Hayward-McClelland SF, Petrin DP, Garber GE. Treatment of infections caused by metronidazole-resistant Trichomonas vaginalis. Clin Microbiol Rev 2004; 17: 783793.

5. Xie YT, Gao JM, Wu YP, Tang P, Hide G, Lai DH, Lun ZR. Recombinant $\alpha$-actinin subunit antigens of Trichomonas vaginalis as potential vaccine candidates in protecting against trichomoniasis. Parasit Vectors 2017; 10: 83.

6. Paintlia MK, Kaur S, Gupta I, Ganguly NK, Mahajan RC, Malla N. Specific IgA response, T-cell subtype and cytokine profile in experimental intravaginal trichomoniasis. Parasitol Res 2002; 88: 338-343.

7. Rosinha GM, Freitas DA, Miyoshi A, Azevedo V, Campos E, Cravero SL, Rossetti O, Splitter G, Oliveira SC. Identification and characterization of a Brucella abortus ATP-binding cassette transporter homolog to Rhizobium meliloti ExsA and its role in virulence and protection in mice. Infect Immun 2002; 70: 50365044.

8. Garmory HS, Titball RW. ATP-binding cassette transporters are targets for the development of antibacterial vaccines and therapies. Infect Immun 2004; 72: 6757-6763.

9. Jomaa M, Yuste J, Paton JC, Jones C, Dougan G, Brown JS. Antibodies to the iron uptake $\mathrm{ABC}$ transporter lipoproteins PiaA and PiuA promote opsonophagocytosis of Streptococcus pneumoniae. Infect Immun 2005; 73: 6852-6859.

10. Wilkinson TC, Gardener MJ, Williams WA. Discovery of functional antibodies targeting ion channels. J Biomol Screen 2015; 20: 454-467.

11. Clapham DE. TRP channels as cellular sensors. Nature 2003; 426: 517-524.

12. Prole DL, Taylor CW. Identification of intracellular and plasma membrane calcium channel homologues in pathogenic para- sites. PLoS One 2011; 6: e26218.

13. Han H, Yi F. New insights into TRP channels: Interaction with pattern recognition receptors. Channels (Austin) 2014; 8: 13-19.

14. Lee KJ, Wang W, Padaki R, Bi V, Plewa CA, Gavva NR. Mouse monoclonal antibodies to transient receptor potential ankyrin 1 Act as antagonists of multiple modes of channel activation. J Pharm Exp Ther 2014; 350: 223-231.

15. Fernández KG, Alvarez-Sánchez ME, Arana-Argáez VE, AlvarezSánchez LC, Lara-Riegos JC, Torres-Romero JC. Genome-wide identification, in silico characterization and expression analysis of ZIP-like genes from Trichomonas vaginalis in response to Zinc and Iron. Biometals 2017; 30: 663-675.

16. Arana-Argáez VE, Chan-Zapata I, Canul-Canche J, FernándezMartín K, Martín-Quintal Z, Torres-Romero JC, Coral-Martínez TI, Lara-Riegos JC, Ramírez-Camacho MA. Immunosuppresive effects of the methanolic extract of Chrysophyllum cainito leaves on macrophage functions. Afr J Tradit Complement Altern Med 2016; 14: 179-186.

17. Stagg AJ, Burke F, Hill S, Knight SC. Isolation of mouse spleen dendritic cells. Methods Mol Med 2001; 64: 9-22.

18. Abraham MC, Desjardins M, Filion LG, Garber GE. Inducible immunity to Trichomonas vaginalis in a mouse model of vaginal infection. Infect Immun 1996; 64: 3571-3575.

19. Smith JD, Garber GE. Trichomonas vaginalis infection induces vaginal $\mathrm{CD} 4{ }^{+} \mathrm{T}$-Cell infiltration in a mouse model: a vaccine strategy to reduce vaginal infection and HIV Transmission. J Infect Dis 2015; 212: 285-293.

20. Han IH, Goo SY, Park SJ, Hwang SJ, Kim YS, Yang MS, Ahn MH, Ryu JS. Proinflammatory cytokine and nitric oxide production by human macrophages stimulated with Trichomonas vaginalis. Korean J Parasitol 2009; 47: 205-212.

21. Koo SJ, Chowdhury IH, Szczesny B, Wan X, Garg NJ. Macrophages promote oxidative metabolism to drive nitric oxide generation in response to Trypanosoma cruzi. Infect Immun 2016; 84: 3527-3541.

22. Filardy AA, Costa-da-Silva AC, Koeller CM, Guimarães-Pinto K, Ribeiro-Gomes FL, Lopes MF, Heise N, Freire-de-Lima CG, Nunes MP, DosReis GA. Infection with Leishmania major induces a cellular stress response in macrophages. PLoS One 2014; 9: e85715.

23. Nemati M, Malla N, Yadav M, Khorramdelazad H, Jafarzadeh A. Humoral and T cell-mediated immune response against trichomoniasis. Parasite Immunol 2018; 40: e12510.

24. Park GC, Ryu JS, Min DY. The role of nitric oxide as an effector of macrophage-mediated cytotoxicity against Trichomonas vaginalis. Korean J Parasitol 1997; 35: 189-195.

25. Murai M, Turovkaya O, Kim G, Madan R, Karp CL, Cheroutre H, Kronenberg M. Interleukin 10 acts on regulatory T cells to maintain expression of the transcription factor Foxp3 and suppressive function in mice with colitis. Nat Immunol 2009; 10: 11781184. 